\title{
Drusen Quantification for Early Identification of Age Related Macular Degeneration
}

\author{
${ }^{1}$ Kajal Kumari and ${ }^{2}$ Deepti Mittal \\ ${ }^{1,2}$ Electrical and Instrumentation Engineering Department, Thapar University, Patiala (Punjab), India; \\ kajalkumari0503@gmail.com; deepti.mittal@thapar.edu
}

\begin{abstract}
Age-related macular degeneration (AMD) is a degenerative disorder in people of age 50 and above, in developed nations, characterized on grading of color fundus images by the presence of pathologies such as drusen in macular area. Currently, there is no treatment which can cure irreversible blindness due to age-related macular degeneration. Therefore, the only feasible option is to prevent the incidence of age-related macular degeneration and avoid this unnecessary vision loss. This paper presents an automated method for early diagnosis of AMD by quantifying drusen on the basis of its size, number and area in macular region from standard color retinal images. Previously used methods, generating unsatisfactory results in some cases, are time consuming, complex and prone to error. Therefore, this paper provides a simple drusen detection and quantification method to detect the exact number of drusen, area and size as well as classify drusen into small, intermediate and soft or large which will further help in initial screening of early stage of age-related macular degeneration and its progression i.e. change in drusen area. The proposed method achieved $93.2 \%$ accuracy for drusen detection and $91.8 \%$ accuracy in small drusen, $98.66 \%$ in intermediate drusen and $92.91 \%$ in soft drusen quantification in order to grade the severity of AMD which outwits the other methods.
\end{abstract}

Keywords: Age-related macular degeneration, pixel-wise feature extraction, drusen subtypes, quantification, fundus images.

\section{Introduction}

Age-related macular degeneration (AMD) is a degenerative disorder of the central area of the retina called macula often associated with visual impairment which is more frequent after 65 years of age in the western world [1-3]. Currently, approximately $35 \%$ of adults older than 80 years in the United States are affected by vision loss which is expected to increase approximately 1.5 fold over the next ten years [5]. AMD can be broadly divided into dry and wet [3,4]. Dry AMD is caused by the lack of functioning of visual cells and generally associated with lesion named drusen and pigmentary changes in the retina [6, 7]. It is also called as early and intermediate AMD and generally associated with only minimal visual symptoms. Late AMD has two types, first one is progressive geography atrophy in the macular region called as late "dry AMD" and second one is an exudative AMD due to development of choroidal neovascularization called as "wet AMD". Wet AMD progresses rapidly and may respond to laser treatment in early stages [6, 8-9]. Most people with macular degeneration are affected by the dry type and there is no treatment for it [10]. Age-Related Eye disease Study (AREDS) showed that the specific anti-oxidant vitamin supplementation reduces the risk of progression from intermediate stage to late AMD stage that can allow for preventive strategies. Currently, there is no cure for AMD, however early detection and subsequent treatment may prevent the severe vision loss or slow the 
progression of the disease [11]. Further strategies are developed to control the risk of progression of AMD from intermediate stage to late. To control the risk of progression it will be important to identify the group of people with early signs so that they should be treated at time by providing treatment trials and user interventions. Therefore, identifying people with early signs of the disease and then determining their risk based upon fundus characteristics is more important from social and economic point of view.

Drusen represent the hallmark of non-exudative or dry AMD and considered as one of the first clinical sign of AMD. These are pale yellow deposits of cholesterol and other materials beneath the Retinal pigment epithelium (RPE), identified by the manual evaluation of retinal fundus images by trained clinicians. Many people over the age of 60 will have some drusen which can be classified as hard or soft depending upon their size [12-13]. Hard drusen are small lesions with sharp borders and soft drusen are those with indistinct borders. Soft drusen has been recognized as precursors to advanced AMD. The international Classification and Grading System for Age Related Maculopathy defines hard drusen as having diameters up to 63 microns, intermediate drusen between 63-125 microns and soft drusen which are greater than 125 microns [14]. Existing AMD grading systems require human grader's subjective manual interaction, which is time consuming, prone to inaccuracy, poor repeatability if experienced graders are not used and difficult to maintain intra or inter-rater agreement. Although a number of automated methods have been developed to classify and quantify drusen after detection as explained below with some limitations.

Brandon and Hoover [15] developed a multi-level method to detect drusen by applying waveletbased feature extraction and Feed Forward Neural Network (FFNN) classifier. It classifies images as normal, large few drusen, large many drusen, fine few drusen, fine many drusen and abnormal drusen. This method did not consider any soft and hard drusen, and distinct and indistinct drusen classification which is an essential step for early detection and grading of AMD. Barriga et al. [16] proposed a method for lesion phenotyping on AMD which will further helped in developing an automated AMD grading system. Amplitude modulation-Frequency modulation (AM-FM) is used to generate multi-scale features for classifying pathological structures such as vessels, retinal background, hard and soft drusen. It was unable to classify distinct and indistinct drusen which is one of the important criteria for AMD grading. Agurto et al. [17] described and evaluated the performance of AM-FM algorithm which was earlier used by Barriga et al. [16]. This algorithm was used to report on the presence and absence of AMD and also successful in detecting the area of drusen but unable to count the number of drusen present in an image. Prasath and Ramaya [18] proposed an automated method for drusen detection and grading of AMD based on GLCM based textural features extraction. This method classified drusen into small, medium and large but some of the samples are misclassified as small drusen. Soliz et al. [19] developed a quantitative assessment method for maculopathy using colour retinal images. They used Gaussian smoothing and probability based thresholding method to quantify drusen and assess longitudinal changes in retinal images presenting with maculopathy following Wisconsin Age-related Maculopathy Degeneration Grading Scheme. A series of manual control tools are provided for a grader to further improve the segmentation results. However, the agreement between the graders on different drusen sizes was only $67 \%$. Smith et al. [20] developed a quantitative assessment method for detecting drusen and its size but unable to grade the severity of AMD. Kumari and Mittal [21] proposed a method for automatic drusen detection and quantification using retinal fundus images. This method was able to calculate the area and number of drusen but did not calculate the size of drusen. Hijazi et al. [22] developed a method which is based on spatial histogram and Case base reasoning approach for classifying image into AMD and non-AMD on the basis of drusen only. It did not calculate size and 
Kajal Kumari and Deepti Mittal; Drusen Quantification for Early Identification of Age Related Macular Degeneration. Advances in Image and Video Processing, Volume 3 No 3, June (2015); pp: 28-40

area of drusen which was a major step for grading the severity of AMD. Mora et al. [23] proposed a method for automatic drusen detection and quantification using retinal images. This method was able to calculate the size and area of segmented drusen but validated only on twenty-two retinal images. Bhuiyan et al. [24] proposed a method for drusen area quantification and also classified drusen into intermediate and soft but unable to quantify drusen on the basis of area.

In summary, the limitations of previous works on drusen detection for AMD grading are the classification of drusen according to its size and insufficient information regarding quantification of drusen. Therefore in the present work, a method for AMD grading is proposed by (i) finding boundary of drusen using thresholding based segmentation, (ii) classifying drusen into small, intermediate and large or soft and (iii) quantify drusen on the basis of size, area and number. The method, designed in this way, showing comparatively higher accuracy than the other methods, accurate quantification of drusen with $91.8 \%$ accuracy for small drusen, $98.66 \%$ for intermediate drusen and $92.91 \%$ for soft drusen quantification, further contributed to diagnose the AMD.

Table 1. Summary of previous research work related to grading of AMD using retinal fundus images.

\begin{tabular}{|c|c|c|c|c|c|}
\hline Method & Year & Specialty & $\begin{array}{l}\text { Image processing } \\
\text { techniques }\end{array}$ & Level of Quantization & Accuracy (\%) \\
\hline $\begin{array}{c}\text { Brandon and } \\
\text { Hoover [15] }\end{array}$ & 2003 & Texture based & $\begin{array}{c}\text { Mexican hat wavelet and } \\
\text { Feed Forward Neural } \\
\text { Network }\end{array}$ & $\begin{array}{l}\text { Drusen or non-drusen } \\
\text { Small or large drusen }\end{array}$ & $\begin{array}{l}87 \\
71\end{array}$ \\
\hline $\begin{array}{l}\text { Barriga et al. } \\
\qquad[16]\end{array}$ & 2009 & Texture based & $\begin{array}{l}\text { Multiscale feature through } \\
\text { Amplitude modulation- } \\
\text { Frequency modulation and } \\
\text { ROI by partial Least square. }\end{array}$ & $\begin{array}{l}\text { Drusen and non-drusen } \\
\text { Soft and hard drusen }\end{array}$ & $\begin{array}{l}\text { Up to } 100 \\
96\end{array}$ \\
\hline $\begin{array}{c}\text { Agurto et al. } \\
\text { [17] }\end{array}$ & 2011 & Texture based & Same as Barriga et al. & Drusen area & 73 \\
\hline $\begin{array}{l}\text { Prasath and } \\
\text { Ramaya [18] }\end{array}$ & 2015 & Texture based & $\begin{array}{l}\text { Morphological operators, } \\
\text { circular hough transform, } \\
\text { texture descriptors }\end{array}$ & $\begin{array}{l}\text { Classified drusen into small, } \\
\text { medium and large }\end{array}$ & 98.5 \\
\hline Soliz et al. [19] & 2002 & $\begin{array}{l}\text { Thresholding } \\
\text { based }\end{array}$ & $\begin{array}{c}\text { Median filtering, Gaussian } \\
\text { smoothing and probability } \\
\text { based thresholding }\end{array}$ & $\begin{array}{c}\text { Drusen and non drusen } \\
\text { Drusen size }\end{array}$ & $\begin{array}{l}71 \\
67\end{array}$ \\
\hline $\begin{array}{l}\text { Smith et al. } \\
{[20]}\end{array}$ & 2003 & $\begin{array}{c}\text { Thresholding } \\
\text { based }\end{array}$ & $\begin{array}{c}\text { Otsu and intensity based } \\
\text { thresholding }\end{array}$ & $\begin{array}{c}\text { Classified drusen into small, } \\
\text { intermediate and large }\end{array}$ & 67 \\
\hline $\begin{array}{l}\text { Kumari and } \\
\text { Mittal [21] }\end{array}$ & 2015 & $\begin{array}{l}\text { Thresholding } \\
\text { based }\end{array}$ & $\begin{array}{l}\text { Illumination correction, } \\
\text { Otsu's thresholding, } \\
\text { morphological operation, } \\
\text { pixel-wise feature extraction }\end{array}$ & Drusen area detection & 96 \\
\hline $\begin{array}{l}\text { Hanafi et al. } \\
\text { [22] }\end{array}$ & 2010 & Cluster based & $\begin{array}{l}\text { Spatial histogram and } \\
\text { similarity based } \\
\text { classification }\end{array}$ & $\begin{array}{l}\text { AMD and non AMD } \\
\text { classification }\end{array}$ & 77 \\
\hline $\begin{array}{l}\text { Mora et al. } \\
\qquad[23]\end{array}$ & 2011 & $\begin{array}{l}\text { Edge and } \\
\text { template based }\end{array}$ & $\begin{array}{c}\text { Illumination correction, } \\
\text { normalization and Gaussian } \\
\text { derivative }\end{array}$ & $\begin{array}{l}\text { Drusen area detection } \\
\text { Drusen pixel detection } \\
\text { sensitivity and specificity }\end{array}$ & $\begin{array}{l}43 \\
68 \\
96\end{array}$ \\
\hline $\begin{array}{l}\text { Bhuiyan et al. } \\
\qquad[24]\end{array}$ & 2013 & $\begin{array}{l}\text { Based on region } \\
\text { growing }\end{array}$ & $\begin{array}{l}\text { Gaussian derivative and } \\
\text { region growing technique }\end{array}$ & $\begin{array}{l}\text { Quantified drusen area } \\
\text { Classified drusen into } \\
\text { intermediate and soft }\end{array}$ & 82.14 \\
\hline
\end{tabular}




\section{Material and method}

\section{Materials}

Database is a tool for evaluation and comparisons of different algorithms. In order to evaluate algorithms for automated screening and diagnosis of retinal disease, some of the benchmark databases are publicly available. The purpose of these databases is to check the validity of systems and to compare the results with the existing techniques. In this paper, two databases named STARE (Structured Analysis of Retina) and ARIA (Automated Retinal Image Analysis) are used for grading the severity of $A M D$ and to compare the results with the existing methods.

The STARE database is acquired using TOPCON fundus camera with 35 degree field of view and a resolution of $700 \times 605$ pixels. It consists of total 400 images form which 36 is for normal AMD and 48 for AMD images [25]. The ARIA database is acquired using Carl Zeiss Meditec fundus camera with 50 degree field of view and a resolution of $768 \times 576$ pixels. It consists of 101 normal and 11 AMD images [26] as described in Table 2.

Table 2 Description of database used in the present work.

\begin{tabular}{|c|c|c|c|c|c|c|}
\hline Database & Camera & $\begin{array}{l}\text { Field of } \\
\text { view }\end{array}$ & Resolution & $\begin{array}{l}\text { No. of } \\
\text { Images }\end{array}$ & $\begin{array}{l}\text { Normal } \\
\text { Images }\end{array}$ & $\begin{array}{c}\text { Images } \\
\text { with AMD }\end{array}$ \\
\hline STARE & TOPCON & 35 degree & $700 \times 605$ & 400 & 36 & 25 \\
\hline ARIA & $\begin{array}{l}\text { Carl Zeiss } \\
\text { Meditec }\end{array}$ & 50 degree & $768 \times 576$ & 450 & 101 & 11 \\
\hline
\end{tabular}

\section{Method}

The proposed method to grade the severity of AMD consists of three phases named as (i) boundary extraction of drusen (ii) quantification of drusen (iii) grading of AMD severity which is discussed below.

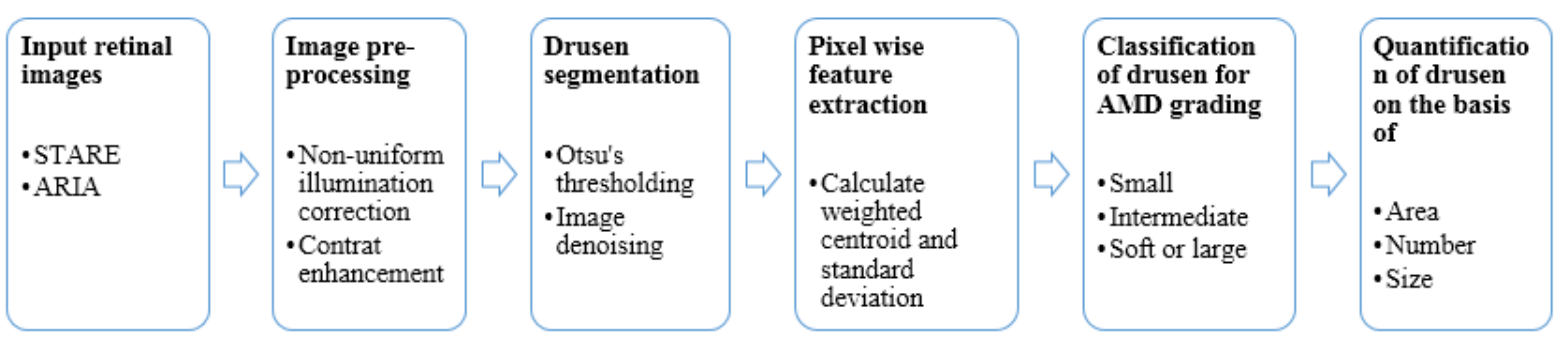

Figure 1. Flow chart of proposed drusen quantification method.

\subsection{Boundary extraction of drusen}

To extract the boundary of drusen following steps are carried out: firstly the retinal fundus images are preprocessed by selecting green channel for better contrast, non-uniform illumination is corrected by subtracting an approximated image from original image then resultant image is enhanced by using automatic contrast enhancement technique. Drusen is segmented using Otsu's thresholding and unwanted noise is removed by morphological operation. Edge of drusen is detected by using canny edge detector [21]. 


\subsection{Drusen detection}

After detecting the edge of drusen, the size, shape and area of each region is analyzed which will further help in grading the severity of AMD. A label matrix is used by Kumari and Mittal [21] to visualize the drusen as a pseudo color indexed image to highlight the drusen area. Position of each drusen is located by using weighted centroid of each region and to highlight the exact number of drusen pixel-wise feature is extracted so that if any drusen is overlapped over other then it can be separate out. Hence the present method provides a platform to diagnose the AMD by locating its position, total number of drusen present in an image as well as by calculating its size and area which is further discussed.

\subsubsection{Drusen shape analysis}

A drusen is circular or oval shaped region. These shapes are 2-D and for verification whether it is circular or oval, boundary detection plays an important role. In this paper shape is analyzed as circular or oval by visualizing the boundary of drusen. The centroid and boundary point of detected drusen is calculated which will help in computing the ratio of minimum and maximum radius to determine the shape as circular or oval.

\subsubsection{Drusen size analysis}

After shape analysis, drusen are filtered on the basis of size. Since all drusen are of circular or oval shaped, the diameter of the region in pixels provides the size of the drusen.

\subsubsection{Quantification of drusen area}

The true area is calculated based on the drusen boundary and region information from the image which includes drusen. To compute the area of drusen, total numbers of pixels are counted as given in Eqn. (1).

$$
\text { Area, } \quad A_{i}=\sum_{r=0}^{H-1} \sum_{c=0}^{W-1} I_{i}(r, c)
$$

The area $A_{i}$ is measured in pixels and it indicates the relative size of the object.

\subsubsection{Number of drusen}

To count the exact number of drusen present in an image pixel wise feature is extracted by calculating the weighted centroid and standard deviation of each object i.e. drusen using pixel value of grey scale image. It is also useful in locating the exact position of each spot that has been blurred over an image region during image acquisition process. This will also help in decomposition of overlapped objects into individual particle []. Hence one can easily count the exact number of drusen present in a given image.

\subsection{Grading of AMD severity}

Retinal color fundus imaging has been widely used for AMD grading. A number of grading protocols has been established for drusen grading which helps in identifying the stage of AMD using color fundus imaging. Among these protocols Wisconsin Age-related Maculopathy Grading system, the International Classification and Grading System for Age-related Maculopathy and Age-related Macular Degeneration and its modified version are widely used [27, 14, 28]. These methods mainly use computer software tools that a grader uses to manually count the drusen through drawing different sizes of circles in the software interface. The quantification of drusen and grading of AMD using manual methods over fundus photographs is not commonly used as it is a fastidious process and lacks reproducibility. Also, the variability within the analyses performed by different 
ophthalmologists limits the use of this technique. Hence, automated AMD grading techniques are used for the diagnosis of disease severity level. There are a number of articles have been published on drusen quantification using retinal fundus imaging but out of these only a few of them are utilized drusen size, area and actual number in the image to grade the AMD severity as explained in the literature review section as well as only a few of techniques can classify into small, intermediate and soft. Most of these automatic techniques are constrained in determining the drusen segmentation level and absence and presence of drusen in the retina.

In this paper, an automatic, accurate and efficient classification and quantification of drusen with considering drusen position, size, shape, area and actual number of drusen present in retinal fundus images can provide a useful tool to define the severity of AMD. Use of such tool would help to determine the severity of risk for progressive sight threatening advanced AMD. AREDS grading system defines the AMD category as 1 to 4 - normal to severe AMD based on the drusen, geography atrophy, retinal pigment epithelium and neovascularization following Wisconsin grading protocol which is applied in this paper. The study defines AMD category as 1 if there is no drusen exists or drusen size < $63 \mu \mathrm{m}$ and total area covered by drusen is $<125 \mu \mathrm{m}$. AMD category 2 or intermediate is defined as drusen size $\geq 63 \mu \mathrm{m}$ and $<125 \mu \mathrm{m}$ and drusen area $\geq 125 \mu \mathrm{m}$ but GA absent. AMD category 3a is defined as intermediate $\geq 63 \mu \mathrm{m}$ and $<125 \mu \mathrm{m}$, drusen area $360 \mu \mathrm{m}$ diameter circle if soft distinct drusen are present and $\geq 656 \mu \mathrm{m}$ diameter circle if soft indistinct drusen are present as illustrated in Table 3 [29].

\begin{tabular}{|c|c|c|c|c|}
\hline & \multicolumn{3}{|c|}{ First Eye } & Second Eye \\
\hline $\begin{array}{l}\text { AMD } \\
\text { Category }\end{array}$ & Drusen size & Drusen Area & Pigment Abnormalities & \\
\hline 1 & None or small $(<63 \mu m)$ & $\begin{array}{c}<125 \mu m \text { diameter circle } \\
\text { (5-15 small } \\
\text { drusen) }\end{array}$ & None & Same as first \\
\hline 2 & $\begin{array}{c}\text { Small }(<63 \mu \mathrm{m}) \text { or } \\
\text { intermediate }(\geq 63, \\
>125 \mu \mathrm{m}) \\
\text { Doesn't matter if pigment } \\
\text { abnormalities present. }\end{array}$ & $\begin{array}{l}\geq 125 \mu \mathrm{m} \text { diameter circle. } \\
\text { At least one drusen. }\end{array}$ & $\begin{array}{l}\text { Absent or present, but } \\
\text { GA absent. }\end{array}$ & $\begin{array}{l}\text { Same as first or } \\
\text { category } 1 .\end{array}$ \\
\hline $3 a$ & $\begin{array}{c}\text { Intermediate }(\geq,<125 \mu \mathrm{m}) \text { or } \\
\text { Large } \\
(\geq 125 \mu \mathrm{m}) . \\
\text { None required if non central } \\
\text { GA present. }\end{array}$ & $\begin{array}{c}\geq 360 \mu \mathrm{m} \text { diameter circle if } \\
\text { soft indistinct drusen are } \\
\text { present ( }=20 \text { intermediate } \\
\text { drusen). } \\
\geq 656 \mu \mathrm{m} \text { diameter circle if } \\
\text { soft indistinct drusen are } \\
\text { absent. } \\
\text { At least one drusen should } \\
\text { present. }\end{array}$ & $\begin{array}{l}\text { Absent or present but } \\
\text { GA absent. }\end{array}$ & $\begin{array}{l}\text { Same as first or } \\
\text { category } 1 \text { or } 2 \text {. }\end{array}$ \\
\hline $3 b$ & $\begin{array}{l}\text { First eye same category as } \\
\text { 3a. }\end{array}$ & & & $\begin{array}{l}\text { Visual Acuity }<20.32 \\
\text { due to AMD; or } \\
\text { unicolor disqualifying } \\
\text { disorder present. }\end{array}$ \\
\hline $4 a$ & $\begin{array}{l}\text { First eye same category as } 1, \\
2 \text { or } 3 a .\end{array}$ & & & Advanced AMD. \\
\hline $4 b$ & $\begin{array}{l}\text { First eye same category as } 1, \\
2 \text { or } 3 a .\end{array}$ & & & $\begin{array}{l}\text { Visual Acuity }<20 / 32 \\
\text { due to AMD, but } \\
\text { advanced AMD not } \\
\text { present. }\end{array}$ \\
\hline
\end{tabular}




\section{Evaluation method}

In this paper, detected drusen with quantification by proposed method is compared with manually graded retinal images. Thirty four images from STARE and ARIA datasets are randomly selected with variety of drusen types. The performance is measured in different steps:

\subsection{Drusen detection accuracy}

It presents the percentage of retinal images with the presence or absence of drusen detected correctly. Segmented drusen from proposed method are compared with ground truth in terms of sensitivity, specificity, accuracy, positive predictive value (PPV) [30-31]. These performance parameters are computed considering drusen pixels positive and background pixels negative which are discussed below. Here $T_{P}$ are true positive means drusen regions correctly classified or number of overlapped positive pixels in drusen and ground truith images. $T_{N}$, are true negative means non drusen regions correctly classified. $F_{P}$, ar false positives means non drusen regions wrongly classified as drusen regions. $F_{N}$, are false negatives means drusen regions wrongly classified as non drusen regions.

$$
\begin{gathered}
\text { Sensitivity }=\frac{T_{P}}{\left(T_{P}+T_{N}\right)} \\
\text { Specificity }=\frac{T_{N}}{\left(T_{N}+F_{P}\right)} \\
\text { Accuracy }=\frac{\left(T_{N}+T_{P}\right)}{\left(T_{N}+T_{P}+F_{N}+F_{P}\right)} \\
\text { PPV }=\frac{T_{P}}{\left(T_{P}+F_{P}\right)}
\end{gathered}
$$

\subsection{Non-overlapped drusen area}

To measure the non-overlapped area, comparison of segmented drusen by proposed method and same with ground truth is done. Here comparison is done pixel-by-pixel to find the true size and area of drusen.

\subsection{Numbers of drusen}

To measure the accuracy of drusen types thirty four images are selected which includes small, intermediate and soft or large drusen. Afterwards, numbers of each drusen type in the individual images are counted for both manually measured and automatically detected. Here accuracy is computed in percentage for different drusen types and also compared with other existing methods.

\section{Results and discussion}

The proposed method for classification and quantification of drusen is implemented in MATLAB version 7.10 on a PC with Intel core i3 $(2.40 \mathrm{GHZ})$ processor. This method is applied on 36 images, publically available datasets i.e. STARE and ARIA, acquired by the fundus camera to diagnose the severity of disease. In this study, automated detected drusen by Kumari, Mittal [21] is compared with manually graded retinal fundus images i.e. ground truth. The performance is evaluated by measuring the drusen detection sensitivity, specificity, accuracy and positive predictive value (PPV) of $82 \%$, $91.3 \%, 93.2 \%$ and $92.21 \%$ respectively as absent or present of drusen in the image.

\subsection{Boundary detection of drusen}

To detect the boundary of drusen, green channel is selected from an image for better contrast and non-uniform illumination is corrected by subtracting approximated image from an original image. 
Afterwards, image with uniform illumination is carried out to obtain more information in terms of better contrast with less noise by using automated contrast enhancement technique and morphological operation respectively [32-33]. Further, drusen is segmented by using Otsu's thresholding and boundary is detected by using conventional canny edge detector as described in method of Kumari and Mittal [21]. Fig. 2 represents the boundary of detected drusen by Kumari and Mittal [21].

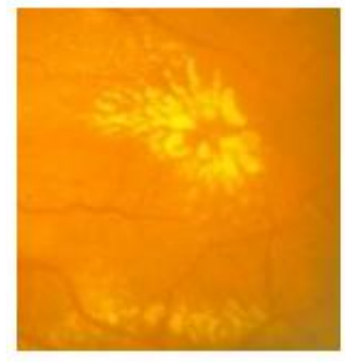

(a)

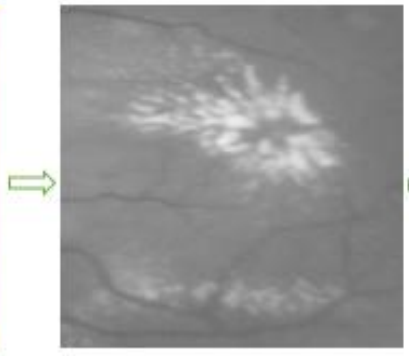

(b)

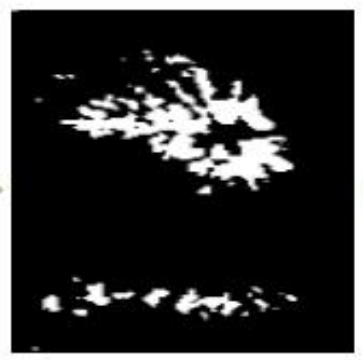

(c)

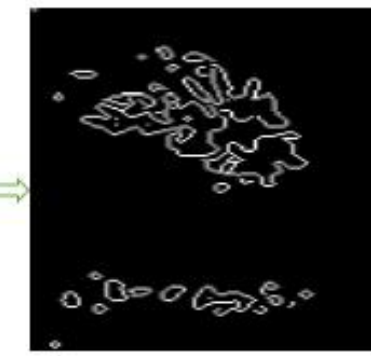

(d)

Figure 2. Result of boundary detectioon of drusen: (a) original image (b) green channel of image, (c) segmented drusen, (d) boundary of detected drusen.

\subsection{Classification and quantification of drusen}

In this paper drusen are classified as small, medium and large on the basis of AREDS categories and quantification is done according to drusen area, size and number present in an image as given in Table 4. Here area is obtained by computing the overall drusen area covered in the macular region by summing the individual drusen area and size is measured in terms of drusen diameter. These values are then converted into micrometers in order to be comparable between images with different resolutions. Here 1 pixel=7microns is used to convert the pixels into microns.

\subsection{Performance measure}

After quantification of segmented drusen it is compared with the ground truth i.e. manually segmented drusen as shown in table 4. Proposed method has achieved $93.2 \%$ drusen detection accuracy, $82 \%$ sensitivity, $91.3 \%$ specificity and $92.21 \%$ PPV. After comparing results of proposed method with ground truth it can be concluded that the present system having better results with less non-overlapped drusen region ranging from 0 to 3.156 for small drusen, 0 to 14.329 for intermediate drusen and 0.72 to 19.41 for large or soft druse. It can be clearly seen from the table 5 , the proposed method achives average accuracy of $91.8 \%$ for small drusen, $98.66 \%$ for intermediate drusen and 92.91\% for large or soft drusen, outwits the results of Bhuiyan et al. [24]. The results demonstrated that the proposed system could identify all the intermediate drusen and took less time of 0.042 seconds to quantify the drusen area.

\section{Conclusion}

Previously, several methods are implemented to segment, classify and quantify the drusen area to measure the severity of AMD in retinal fundus images. This direct method are limited due to user involvement for ROI selection, varying image conditions and lack of adaptive capabilities of the methods, results in poor segmentation and quantification such as over or under segmentations in some parts of the image as well as it requires large computational effort. Therefore, an early treatment and effective planning by using computerized method is highly reliable and may reduce the progression of disease and also provide timely treatment after quantifying drusen which helps in grading the severity of AMD. In this work, an automated segmentation approach has been developed 
Kajal Kumari and Deepti Mittal; Drusen Quantification for Early Identification of Age Related Macular Degeneration. Advances in Image and Video Processing, Volume 3 No 3, June (2015); pp: 28-40

to classify and quantify drusen for grading the severity of AMD using publicly available retinal fundus images. It analyzes region properties of image by extracting the feature from the pixels of fundus images which will help in decomposition of overlapped objects to evaluate the exact number of drusen. This method will help in computing the total area affected by drusen in pixels with its size, shape and number present in an image. The proposed method outperforms the other existing method by achieving $82 \%, 91.3 \%, 93.2 \%$ and $92.21 \%$ for sensitivity, specificity, accuracy and PPV respectively in addition with accuracy of $991.8 \%$ a for small drusen, $98.66 \%$ for intermediate drusen and $92.91 \%$ for large or soft drusen with less non-overlapping area as compared to ground truth. It can be concluded that the use of the proposed detector may reduce the irreversible blindness across the world and give reliable results in treatment of disease.

Table 4. Classification and Quantitative analysis of drusen to grade the severity of AMD.

\begin{tabular}{|c|c|c|c|c|c|c|c|c|}
\hline & Images & $\begin{array}{l}\text { Total no. } \\
\text { Of drusen } \\
\text { by the } \\
\text { ground } \\
\text { truth }\end{array}$ & $\begin{array}{l}\text { Total drusen area } \\
\text { in the ground } \\
\text { truth }\left(\mu m^{2}\right)\end{array}$ & $\begin{array}{c}\text { Size of } \\
\text { drusen } \\
(\mu m)\end{array}$ & $\begin{array}{l}\text { Total no. } \\
\text { Of drusen } \\
\text { by the } \\
\text { proposed } \\
\text { method }\end{array}$ & $\begin{array}{l}\text { Total drusen area } \\
\text { by the proposed } \\
\text { method } \mu m^{2}\end{array}$ & $\begin{array}{l}\text { Size of } \\
\text { drusen } \\
\text { in } \mu m\end{array}$ & $\begin{array}{c}\text { Non- } \\
\text { overlapp } \\
\text { ed area }\end{array}$ \\
\hline \multirow{7}{*}{ SMALL } & IM0013 & 4 & 73.58 & 46.508 & 4 & 72.85 & 46.508 & 0.00 \\
\hline & IM0015 & 12 & $1.362 \times 10^{11}$ & 39.627 & 10 & $1.0035 \times 10^{11}$ & 36.471 & 3.156 \\
\hline & IM0046 & 15 & 63.84685866 & 98.899 & 12 & 73.84675856 & 98.880 & 0.019 \\
\hline & IM0262 & 9 & $2.028 \times 10^{10}$ & 52.829 & 8 & $1.997 \times 10^{10}$ & 51.064 & 1.765 \\
\hline & IM0401 & 6 & $1.317 \times 10^{10}$ & 48.752 & 6 & $1.188 \times 10^{10}$ & 48.518 & 0.234 \\
\hline & IM0402 & 5 & $1.173481523 \times 10^{10}$ & 25.975 & 5 & $1.021730348 \times 10^{10}$ & 25.135 & 0.84 \\
\hline & Images & $\begin{array}{l}\text { Total no. } \\
\text { Of drusen } \\
\text { by the } \\
\text { ground } \\
\text { truth }\end{array}$ & $\begin{array}{l}\text { Total drusen area in } \\
\text { the ground truth } \\
\left(\mu m^{2}\right)\end{array}$ & $\begin{array}{c}\text { Size of } \\
\text { drusen } \\
(\mu m)\end{array}$ & $\begin{array}{l}\text { Total no. } \\
\text { Of drusen } \\
\text { by the } \\
\text { proposed } \\
\text { method }\end{array}$ & $\begin{array}{l}\text { Total drusen area } \\
\text { by the proposed } \\
\text { method } \mu \mathrm{m}^{2}\end{array}$ & $\begin{array}{l}\text { Size of } \\
\text { drusen in } \\
\quad \mu m\end{array}$ & $\begin{array}{l}\text { Non- } \\
\text { overlapp } \\
\text { ed area }\end{array}$ \\
\hline \multirow{12}{*}{$\begin{array}{l}\text { INTERME } \\
\text { DIATE }\end{array}$} & IM0006 & 35 & $1.973 \times 10^{11}$ & 72.002 & 33 & $1.581 \times 10^{11}$ & 63.446 & 8.556 \\
\hline & IM0007 & 18 & $1.263 \times 10^{11}$ & 68.271 & 18 & $1.240 \times 10^{11}$ & 68.116 & 0.155 \\
\hline & IM0012 & 46 & $1.463 \times 10^{11}$ & 74.002 & 43 & $1.365 \times 10^{11}$ & 62.436 & 11.566 \\
\hline & IM0033 & 87 & $2.033 \times 10^{11}$ & $\begin{array}{c}106.11 \\
1\end{array}$ & 87 & $2.025 \times 10^{11}$ & 104.028 & 2.083 \\
\hline & IM0038 & 25 & $1.168 \times 10^{10}$ & 67.002 & 25 & $1.002 \times 10^{11}$ & 64.363 & 2.639 \\
\hline & IM0066 & 50 & $2.209 \times 10^{10}$ & $\begin{array}{c}103.98 \\
1\end{array}$ & 50 & $2.368 \times 10^{10}$ & 95.652 & 14.329 \\
\hline & IM0068 & 125 & $1.689 \times 10^{10}$ & 63.932 & 125 & $1.653 \times 10^{10}$ & 63.500 & 0.432 \\
\hline & IM0079 & 40 & $1.135 \times 10^{10}$ & 69.842 & 36 & $1.026 \times 10^{11}$ & 69.225 & 0.617 \\
\hline & IM0097 & 20 & $1.139 \times 10^{10}$ & $\begin{array}{c}127.85 \\
4\end{array}$ & 20 & $1.133 \times 10^{10}$ & 127.854 & 0.0 \\
\hline & IM0192 & 136 & $2.603 \times 10^{12}$ & $\begin{array}{c}118.29 \\
5\end{array}$ & 136 & $2.031 \times 10^{12}$ & 118.268 & 0.027 \\
\hline & IM0263 & 89 & $1.759 \times 10^{10}$ & 72.654 & 89 & $1.708 \times 10^{10}$ & 72.652 & 0.002 \\
\hline & IM0376 & 2 & 90.985 & $\begin{array}{c}127.54 \\
1\end{array}$ & 2 & 90.977 & 127.541 & 0.008 \\
\hline
\end{tabular}




\begin{tabular}{|c|c|c|c|c|c|c|c|c|}
\hline \multirow{17}{*}{ 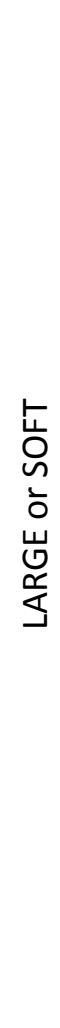 } & Images & $\begin{array}{c}\text { Total no. } \\
\text { Of drusen } \\
\text { by the } \\
\text { ground } \\
\text { truth }\end{array}$ & $\begin{array}{l}\text { Total drusen } \\
\text { area in the } \\
\text { ground truth } \\
\left(\mu m^{2}\right)\end{array}$ & $\begin{array}{c}\text { Size of } \\
\text { drusen } \\
(\mu m)\end{array}$ & $\begin{array}{l}\text { Total no. Of } \\
\text { drusen by } \\
\text { the proposed } \\
\text { method }\end{array}$ & $\begin{array}{l}\text { Total drusen } \\
\text { area by the } \\
\text { proposed } \\
\text { method } \mu m^{2}\end{array}$ & $\begin{array}{c}\text { Size of } \\
\text { drusen in } \mu m\end{array}$ & $\begin{array}{c}\text { Non- } \\
\text { overlapped } \\
\text { area }\end{array}$ \\
\hline & IM0003 & 124 & $3.782 \times 10^{11}$ & 157.241 & 119 & $3.255 \times 10^{11}$ & 157.002 & 0.239 \\
\hline & IM0037 & 45 & $2.511 \times 10^{11}$ & 152.115 & 45 & $2.156 \times 10^{11}$ & 150.221 & 1.894 \\
\hline & IM0041 & 125 & $1.984 \times 10^{11}$ & 92.475 & 120 & $1.664 \times 10^{11}$ & 92.036 & 0.439 \\
\hline & IM0063 & 56 & $3.620 \times 10^{10}$ & 248.708 & 52 & $3.160 \times 10^{10}$ & 248.521 & 0.187 \\
\hline & IM0143 & 27 & $9.484 \times 10^{10}$ & 336.125 & 27 & $9.454 \times 10^{10}$ & 336.020 & 0.105 \\
\hline & IM0147 & 33 & $3.329 \times 10^{11}$ & 183.6320 & 33 & $3.332 \times 10^{11}$ & 183.613 & 0.019 \\
\hline & IM0148 & 41 & $5.113 \times 10^{11}$ & 82.366 & 41 & $5.039 \times 10^{11}$ & 82.285 & 0.081 \\
\hline & IM0265 & 43 & $1.201 \times 10^{10}$ & 176.24 & 40 & $1.386 \times 10^{10}$ & 174.625 & 1.615 \\
\hline & IM0266 & 98 & $6.935 \times 10^{10}$ & 558.68 & 94 & $6.283 \times 10^{10}$ & 539.27 & 19.41 \\
\hline & IM0267 & 112 & $1.496 \times 10^{10}$ & 669.252 & 106 & $1.251 \times 10^{11}$ & 652.395 & 16.857 \\
\hline & IM0270 & 10 & $5.174 \times 10^{10}$ & 152.231 & 10 & $5.135 \times 10^{10}$ & 152.135 & 0.096 \\
\hline & IM0271 & 26 & $7.132 \times 10^{10}$ & 119.565 & 26 & $7.083 \times 10^{10}$ & 119.327 & 0.238 \\
\hline & IM0281 & 31 & $2.219 \times 10^{10}$ & 560.013 & 31 & $2.218 \times 10^{10}$ & 556.916 & 3.097 \\
\hline & IM0288 & 39 & $3.998 \times 10^{11}$ & 146.522 & 36 & $3.081 \times 10^{11}$ & 143.366 & 3.156 \\
\hline & IM0382 & 82 & $2.676 \times 10^{11}$ & 445.22 & 82 & $2.580 \times 10^{11}$ & 444.50 & 0.72 \\
\hline & $\underset{3}{\text { ARIA_1 }}$ & 96 & $5.073 \times 10^{11}$ & 135.236 & 92 & $4.998 \times 10^{11}$ & 132.583 & 2.653 \\
\hline
\end{tabular}

Table 5. Summary of overall accuracy on small, intermediate and soft drusen detection by proposed method and method of Bhuiyan et al. [].

\begin{tabular}{|c|c|c|c|c|c|}
\hline Method & Category & $\begin{array}{c}\text { Number of } \\
\text { images }\end{array}$ & $\begin{array}{c}\text { Total number } \\
\text { of drusen in } \\
\text { ground truth }\end{array}$ & $\begin{array}{c}\text { Number of } \\
\text { drusen } \\
\text { correctly } \\
\text { detected }\end{array}$ & Accuracy (\%) \\
\hline & $\begin{array}{c}\text { Intermediate drusen }(63-125 \\
\mu m)\end{array}$ & 12 & 49 & 39 & 79.59 \\
$\begin{array}{c}\text { [25] } \\
\text { Proposed } \\
\text { method }\end{array}$ & $\begin{array}{c}\text { Soft drusen }(>125 \mu m) \\
\text { Small drusen }(<63 \mu m)\end{array}$ & 6 & 28 & 23 & 82.14 \\
& $\begin{array}{c}\text { Intermediate drusen (63-125 } \\
\mu m)\end{array}$ & 12 & 673 & 664 & 98.66 \\
\hline $\begin{array}{c}\mu \text { Large or soft drusen }(>125 \\
\mu m)\end{array}$ & 16 & 988 & 918 & 92.91 \\
\hline
\end{tabular}

\section{REFRENCES}

[1]. $\quad$ Lim LS, Mitchell P, Seddom JM et al. Age-related macular degeneration.t, 2012. 379: 17281738.

[2]. Bartlett and Eperjesi. use of fundus imaging in quantification of age-related macular degeneration. Surv Ophthalmol, 2007. 52: 655-671. 
[3]. Wong, Liew et al. clinical update: new treatments for age-related macular degeneration. Lancet, 2007. 370: 204-206.

[4]. Wong and Rogers. Statins and age-related macular degeneration: time for a randomized controlled trial? Am J Ophthalmol, 2007. 144: 117-119.

[5]. Age-related Eye Disease Study Research Group. The age-related eye disease study system for classifying age-related macular degeneration from stereoscopic color fundus photographs: the age-related eye disease study report number 6 . 2001;132:668-681.

[6]. De Jong, Age-related macular degeneration. The New England Journal of Medicine, 355(14), 2006. p: 1474-1485.

[7]. Kanagasingam, Bhuiyan et al. Progress on retinal image analysis for age related macular degeneration, Progress in Retinal Eye Research, 2014. 38:20-42.

[8]. Lim, Laude et al. Age-related macular degeneration: an Asian perspective. Ann. Acad. Med. Singapore, 2007. 36 (10), S15.

[9]. Mitchell, Smith, Attebo et al. Prevalence of age-related maculopathy in Australia. The Blue Mountains eye study, Ophthalmology, 1995. 102 (10):1450-1460.

[10]. A.-R.E.D.S.R. Group.A randomized, placebo-controlled, clinical trial of highdose supplementation with vitamins $c$ and $e$, beta carotene, and zinc for agerelated macular degeneration and vision loss: AREDS report no. 8, Arch. Ophthalmology, 2001. 119 (10):1417-1436.

[11]. Wong, Chakravarthy et al. The natural history and prognosis of neovascular agerelated macular degeneration: a systematic review of the literature and metaanalysis. Ophthalmology, 2008. 115: 116-126.

[12]. Chopdar, Chakravarthy et al. Age related macular degeneration.British Medical Journal, 2003. 326 (7387):485-488.

[13]. Mookiah, Acharyaet al. Computer-aided diagnosis of diabetic retinopathy: A review. Computer in Biology and Medicine, 2013. 43 (12):2136-2155.

[14]. Bird, Bressler et al. An international classification and grading system for agerelated Maculopathy and age-related macular degeneration. The International ARM Epidemiological Study Group. Surv Ophthalmol, 1995. 39: 367-374.

[15]. Brandon and Hoover. Drusen Detection in a Retinal Image Using Multi- Level Analysis. LNCS, 2003. 618-625.

[16]. Barriga, Murray, Agurto, Pattichis et al. Multiscale AM-FM for lesion phenotyping on age-related macular degeneration. INSPEC, 2009. p: 1-5. 
[17]. Agurto, Barriga, Murray et al. Automatic detection of diabetic retinopathy and agerelated macular degeneration in digital fundus images. Invest Ophthalmol Vis Sci, 2011. 52: 5862-5871.

[18]. Prasath and Ramaya. Detection of macular drusen based on texture descriptors. Research journal of information technology, 2015. 7 (1): 70-79.

[19]. Soliz, Wilson, Nemeth and Nguyen P. Computer-aided methods for quantitative assessment of longitudinal changes in retinal images presenting with maculopathy. SPIE, 2002. 4681:159-170.

[20]. Smith, Nagasaki, Sparrow et al. A method of drusen measurement based on the geometry of fundus reflectance. Biomedical Engineering, 2003. Online 2: 10.

[21]. Kumari and Mittal. Automated Drusen Detection Technique for Age-Related Macular Degeneration. Journal of biomedical engineering in medical imagiong, 2015. 2:18-26.

[22]. Hanafi, Hijazi, Coenen and Zheng. Retinal Image Classification for the Screening of Age-related Macular Degeneration. In: SGAI International Conference on Artificial Intelligence, 2010. p: 325-338.

[23]. Mora, Vieira, Manivannan and Fonseca. Automated drusen detection in retinal images using analytical modelling algorithms. Biomedical Engineering Online, 2011. p: 10: 59.

[24]. Bhuiyan, Kawasaki, Sasaki et al. Drusen detection and quantification for early identification of Age-related macular degeneration using color fundus imaging. Clinical and experimental ophthalmology, 2013. 4: 305. DOI: 10.4172/21559570.1000305 .

[25]. STARE database. Available at http://www.ces.clemson.edu/ ahoover/stare.

[26]. ARIA database. Available at http://www.evecharity.com/aria online.

[27]. Klein, Devis et al. The Wisconsin age-related Maculopathy grading system. Ophthalmology, 1991. 98: 1128-1134.

[28]. Bartlett and Eperjesi. Use of fundus imaging in quantification of age-related macular change. Surv Ophthalmol, 2007. 52: 655-671.

[29]. Age-Related Eye Disease Study Research Group (1999) The Age-Related Eye Disease Study (AREDS): design implications. AREDS report no. 1. Control Clin Trials 20: 573600.

[30]. Mittal, Kumar et al. Neural Network based focal liver lesion diagnosis using ultrasound images. Computerized Medical Imaging and Graphics, 2011. 35:315323. 
Kajal Kumari and Deepti Mittal; Drusen Quantification for Early Identification of Age Related Macular Degeneration. Advances in Image and Video Processing, Volume 3 No 3, June (2015); pp: 28-40

[31]. Chugh, Kaur and Mittal. Exudates segmentation in retinal fundus images for the detection of diabetic retionapthy. International journal of engineering research and technology, 2014. 3: 673-677.

[32]. Mittal, Kumar, Saxenaet al. Enhancement of ultrasound images by modified diffusion method, Medical and Biological Engineering and Computing, 2010. 48(12): 1281-1291.

[33]. Kaur and Mittal. Segmentation and measurement of exudates in fundus images of the retina for detection of retinal diseases. Journal of biomedical engineering and medical imaging, 2015. 2: 27-38. 